\title{
Avaliação do foto-eletrocardiograma como ferramenta de segunda opinião formativa
}

\author{
Evaluation of the photo-electrocardiogram as a tool for formative second opinion
}

\author{
Ana Paula Finatto Canabarro ${ }^{1}$, Ângelo Marcelo Schwalbert ${ }^{2}$, Ricardo Bertoglio Cardoso ${ }^{3}$, \\ Clarissa Francisca Valdez ${ }^{4}$, Carolina Sturm Trindade ${ }^{5}$, Helena W. de Oliveira ${ }^{6}$, \\ Ingrid G Lamadrid ${ }^{7}$, Thais Russomano ${ }^{8}$
}

Biomédica. Aluna de Extensão em Pesquisa do Centro de Microgravidade (MicroG) - Pontifícia Universidade Católica do Rio Grande do Sul (PUCRS). Porto Alegre, RS.

2 Médico. Aluno de Extensão em Pesquisa do MicroG-PUCRS. Porto Alegre, RS

Engenheiro Eletricista. Coordenador de Pesquisa, Desenvolvimento e Inovação do MicroG-PUCRS

Médica da Força Aérea Brasileira, Quinto Comando Aéreo Regional. Canoas, RS.

${ }_{5}^{5}$ Mestre em Ciências da Computação pela Universidade Federal do Rio Grande do Sul (UFRGS). Professora Assistente do Departamento de

Educação e Informação em Saúde da Universidade Federal de Ciências da Saúde de Porto Alegre (UFCSPA). Porto Alegre, RS

${ }^{6}$ Doutora em Estomatologia Clínica. Coordenadora do Laboratório de Telessaúde, MicroG-PUCRS. Porto Alegre, RS.

Estudante da Faculdade de Engenharia da PUCRS. Auxiliar de Laboratório, MicroG-PUCRS. Porto Alegre, RS.

${ }^{8} \mathrm{PhD}$ em Fisiologia Aeroespacial, Kings College London. Professora da Faculdade de Medicina da PUCRS. Coordenadora do MicroG-PUCRS. Porto Alegre, RS.

\begin{abstract}
RESUMO
Objetivos: Avaliar o foto-eletrocardiograma (foto-ECG), como uma ferramenta de segunda opinião formativa a distância. Métodos: Cinquenta eletrocardiogramas (ECGs) em papel milimetrado foram fotografados duas vezes, a primeira utilizando-se uma câmera digital Canon, na resolução 0,3 megapixel, e a segunda com um celular Nokia com câmera acoplada, na resolução 2,0 megapixels, gerando 100 foto-ECGs. Por meio de estudos-piloto, definiu-se o método de aquisição das imagens. Os 100 foto-ECGs foram randomizados, criptografados e enviados a um cardiologista remoto por e-mail; enquanto os 50 ECGs em papel milimetrado lhe foram entregues pessoalmente, sem randomização. Sexo e idade foram as únicas informações dos pacientes disponibilizadas ao especialista.

Resultados: A análise dos dados demonstrou divergência em 14 dos 50 laudos (28\%) na comparação dos ECGs originais com os foto-ECGs adquiridos pela câmera Canon e de 13 dos 50 laudos (26\%) entre os laudos dos ECGs originais e dos foto-ECGs capturados pelo celular Nokia. Houve concordância considerável (Kappa=0,356) entre as interpretações de foto-ECGs e ECGs em papel, tanto para o celular Nokia quanto para a câmera Canon.

Conclusões: A concordância entre o foto-ECG e os traçados originais demonstrou que o método descrito nesse estudo tem potencial de ser utilizado como uma ferramenta de auxílio à prática clínica, desde que a obtenção dos foto-ECGs seja adaptada de forma a melhorar as imagens dos exames. Apenas com concordância boa a ótima em relação aos ECGs originais, o foto-ECG possibilitará a segunda opinião formativa a distância, conferindo melhores opções diagnósticas e terapêuticas.
\end{abstract}

DESCRITORES: TELEMEDICINA; TELEFONE CELULAR; TÉCNICAS DE DIAGNÓSTICO CARDIOVASCULAR; ELETROCARDIOGRAFIA.

\begin{abstract}
Aims: This study aimed to evaluate the photo-electrocardiogram (photo-ECG), as an alternative tool to enable remote formative second opinion in cardiology.

Methods: Fifty paper electrocardiograms (ECGs) were photographed two times, the first using a Canon digital camera, 0.3 megapixel resolution, and the second using a Nokia mobile phone integrated camera, 2.0 megapixel resolution, resulting in 100 Photo-ECGs. A pilot study was responsible for determining the acquisition method. The 100 Photo-ECGs were randomized, encrypted and sent by e-mail to a remote cardiologist, while the 50 paper ECGs were delivered to him in person, without randomization. Gender and age were the only patient information made available to the specialist. Results: Data analysis demonstrated a disagreement in 14 of 50 interpretations (28\%) when comparing paper ECGs to the Canon camera photo-ECGs and in 13 of 50 interpretations (26\%) when comparing paper ECGs to the Nokia camera photo-ECGs. The Kappa test revealed a fair agreement (Kappa $=0.356)$ between interpretations when comparing the original ECGs to their respective photo-ECGs for both camera devices.

Conclusion: The concordance between photo-ECGs and original tracings demonstrated that the method described herein has the potential for use as a tool to assist clinical practice, provided that the acquisition of photo-ECGs is adapted so as to improve exam images. Only with good to very good concordance between the original ECGs and photo-ECGs will remote formative second opinion be possible, giving better diagnostic and therapeutic options.
\end{abstract}

KEY WORDS: TELEMEDICINE; CELLULAR PHONE; DIAGNOSTIC TECHNIQUES, CARDIOVASCULAR; ELETROCARDIOGRAPHY. 


\section{INTRODUÇÃO}

O eletrocardiograma (ECG) é um exame que utiliza o registro das variações dos potenciais de ação gerados pela atividade elétrica do coração para avaliação cardíaca. ${ }^{1}$ Essa técnica apresenta sensibilidade em torno de $80 \%$ e especificidade de $90 \%,{ }^{2}$ o que o tornou o exame cardiológico mais realizado no campo médico. ${ }^{1}$ Com o surgimento das telecomunicações, novas tecnologias puderam ser agregadas ao exame eletrocardiográfico.

As telecomunicações têm se mostrado uma poderosa ferramenta para ultrapassar as barreiras de tempo e distância. Devido à crescente aquisição de computadores e telefones celulares pela população mundial e à introdução da internet de banda larga na telefonia móvel, já é possível enviar e receber arquivos de dados quando e onde for necessário. ${ }^{3}$ Quando esses recursos tecnológicos são utilizados em conjunto para um objetivo específico, tem-se o conceito de Tecnologia da Informação e Comunicação. ${ }^{4}$ A Telemedicina é o ramo da Medicina que tem como finalidade permitir, por meio da aplicação de Tecnologias da Informação e Comunicação, a assistência remota a comunidades que não possuem cuidados de saúde especializados..$^{5-7}$

A Telemedicina surgiu como uma opção inovadora para a melhoria do diagnóstico e do tratamento médico, podendo evitar a remoção de pacientes para avaliações especializadas. Do ponto de vista clínico, ela permite que os profissionais da saúde ofereçam assistência qualificada, vencendo restrições de caráter geográfico e organizacional. ${ }^{7}$

Dentre as diversas ramificações da Telemedicina, surgiu a Telecardiologia, a qual estuda a aplicação das Tecnologias da Informação e Comunicação na prática cardiológica. ${ }^{8}$ Em particular, a avaliação remota da atividade cardíaca, realizada por meio do tele-eletrocardiograma (tele-ECG), tem recebido maior atenção pela comunidade científica. ${ }^{8}$ Atribui-se esse fato ao alto índice de mortes associadas a doenças cardiovasculares, das quais se podem destacar a isquemia cardíaca, sendo o infarto agudo do miocárdio a ocorrência mais grave. ${ }^{9,10}$ Com a Telecardiologia, objetiva-se evitar o deslocamento desnecessário de pacientes atendidos nas unidades de saúde, diminuindo custos operacionais, o tempo para o atendimento dos pacientes e a superlotação dos hospitais.

Ocorre que as tecnologias digitais disponíveis para a aquisição e transmissão de ECGs ainda possuem alto valor agregado e utilizam equipamentos que dificilmente estão disponíveis em postos de saúde carentes de cardiologistas. ${ }^{1}$ Dessa forma, torna-se imprescindível o desenvolvimento de ferramentas alternativas para aquisição e envio de registros eletrocardiográficos, permitindo a implantação em larga escala desses sistemas.

Fotografar o traçado eletrocardiográfico com um smartphone, câmera digital ou tablet e enviar a imagem (foto-ECG) a um cardiologista remoto é uma das atuais opções para redução desses custos. Essa prática já é utilizada em alguns locais, principalmente naqueles que, além de não possuírem ECGs digitais, carecem de profissionais capacitados para analisar o ECG, ou mesmo quando se busca uma segunda opinião formativa. Entretanto, a realização dessa atividade ainda não é cientificamente comprovada. Caso validada, a opção pelo foto-ECG poderá produzir um impacto positivo na prática médica, visto que viabilizará, com investimentos menores, o envio rápido dos dados cardiológicos de pacientes de uma unidade de saúde remota para análise por profissionais especializados. Como essa técnica possibilita a utilização dos eletrocardiógrafos já presentes nas unidades de saúde, não se faz necessária a compra de novos aparelhos (que possuam sistema integrado de transmissão de dados) ou o treinamento extensivo das equipes, reduzindo-se gastos de implantação e operação do tele-ECG.

No entanto, para a implantação do foto-ECG, é necessário garantir a qualidade do sistema de aquisição e visualização das imagens digitalizadas. As características técnicas de câmeras digitais, monitores de visualização e forma de transmissão devem ser determinadas por meio de estudos e processos de avaliação, de forma a garantir o melhor custo-benefício, mantendo a qualidade das informações disponíveis para diagnóstico.

Assim, o presente estudo buscou avaliar o foto-ECG como uma ferramenta alternativa de segunda opinião a distância de cardiologistas para análise de ECGs.

\section{MÉTODOS}

O presente trabalho foi aprovado pelo Comitê de Ética da Pontifícia Universidade Católica do Rio Grande do Sul, sob o parecer número 369/12. Este é um estudo de natureza tecnológica, transversal, retrospectivo e simples-cego e foi desenvolvido em quatro etapas. Estudos-piloto foram realizados previamente a fim de padronizar a técnica de aquisição de imagens e transmissão de informações com os cardiologistas.

\section{Etapa 1: obtenção dos ECGs}

Cinquenta ECGs em papel milimetrado foram adquiridos do arquivo da Junta de Saúde do Hospital 
de Aeronáutica de Canoas, Rio Grande do Sul. Os traçados foram obtidos sem distinção de gênero, idade ou diagnóstico clínico, provindos de pacientes hígidos e cardiopatas. As únicas informações registradas a respeito desses pacientes foram o sexo e a idade.

\section{Etapa 2: aquisição do foto-ECG}

Para a captura das imagens, foram utilizados uma câmera digital modelo Canon IXUS 220 HS na resolução 0,3 megapixel, e um telefone celular modelo Nokia 5130 com câmera acoplada, na resolução 2,0 megapixels, totalizando 100 foto-ECGs (50 com a câmera e 50 com o celular). $\mathrm{O}$ critério "resolução mínima disponível no equipamento" foi utilizado durante os estudos-piloto para definir quais resoluções seriam configuradas na câmera fotográfica e no celular. Acredita-se que, dessa forma, a técnica possui maior replicabilidade, visto que uma maior quantidade de equipamentos fotográficos disponíveis atualmente no mercado possui essas resoluções e/ou superiores.

Por meio dos estudos-piloto, definiu-se o método de aquisição, que determinou:

1) as características de iluminação do ambiente: luminosidade controlada, provida por 12 lâmpadas fluorescentes de $32 \mathrm{~W}$, uniformemente distribuídas em pares;

2) as configurações das câmeras: (a) câmera fotográfica: foco automático, sem uso de flash, proporção 4:3, compressão fina e temporizador desligado; (b) câmera do celular: modo paisagem, sem efeito, balanço de branco automático e qualidade da imagem alta.

3) o protocolo de captura das imagens: para a fixação do ECG foi utilizada uma prancheta, coberta por uma cartolina preta (Figura 1). Quando utilizada a câmera digital, fez-se uso de um tripé para estabilização do equipamento. $\mathrm{Na}$ aquisição, tanto a câmera como o celular foram posicionados paralelamente à superfície do papel, permitindo que a extensão completa da folha do ECG fosse fotografada. A aquisição das fotografias foi realizada sempre pelo mesmo pesquisador.

\section{Etapa 3: análise do traçado}

Os 100 foto-ECGs foram randomizados por meio do software Research Randomizer ${ }^{\circledR}$, e, juntamente com as informações de sexo e idade do paciente, criptografados por meio do aplicativo Adobe Acrobat $\mathrm{Pro}^{\circledR}$. Os foto-ECGs foram então enviados a um cardiologista remoto (simples-cego).

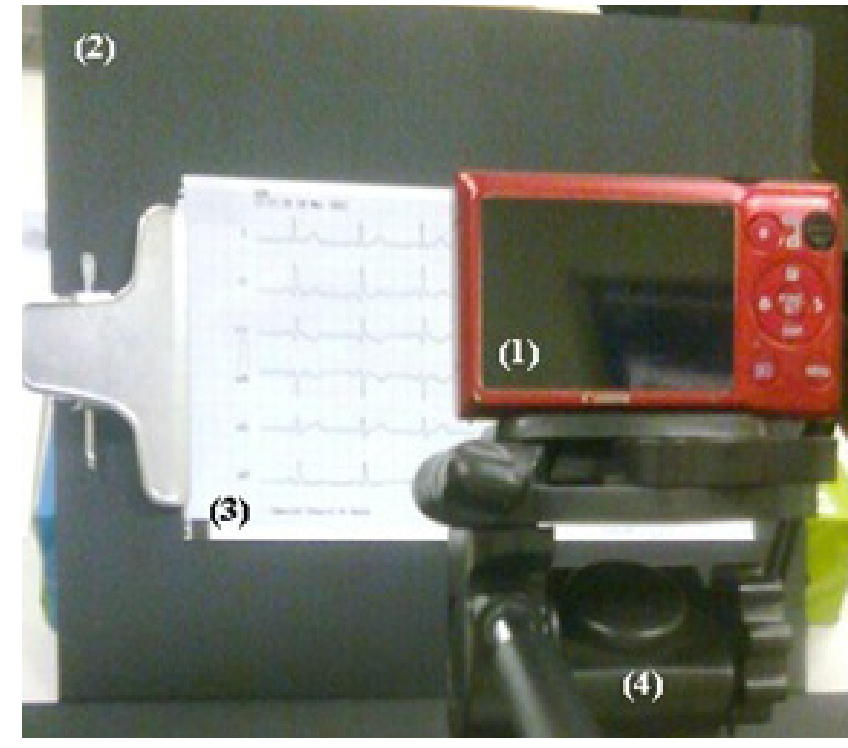

Figura 1. Montagem para aquisição do foto-eletrocardiograma: (1) máquina fotográfica digital; (2) prancheta com cartolina preta; (3) eletrocardiograma em papel milimetrado; (4) tripé.

Os ECGs originais, também com as respectivas informações do paciente, foram entregues pessoalmente ao mesmo cardiologista, com objetivo de evitar o viés de possível discrepância entre ECG original e foto-ECG consequente à interpretação de diferentes profissionais. Para impedir a memorização dos laudos, os ECGs originais foram entregues em momento distinto ao da apresentação dos foto-ECGs, cuidando-se para que o intervalo de entrega não fosse menor que um mês e que o cardiologista não tivesse acesso à sua avaliação prévia para comparação.

De forma a padronizar a análise dos ECGs, foi utilizado pelo cardiologista um protocolo de avaliação, e para cada ECG havia uma ficha contendo os seguintes campos para preenchimento: número do ECG, sexo e idade do paciente, frequência cardíaca, ritmo cardíaco, complexo QRS, intervalos PR e QT, ondas P, Q, R, S e T, segmento ST, presença ou não da onda $U$, eixo e laudo final. Requisitou-se que a(s) derivação(ões) utilizada(s) em cada item fossem anotadas ao lado do valor do mesmo e que o cardiologista apresentasse seu parecer sobre a dificuldade de analisar o exame. Definiu-se que as ondas (exceção da onda U), o complexo QRS e os segmentos deveriam ser registrados em número de quadrados (do papel milimetrado), não necessitando da conversão em milissegundos ou segundos.

Os foto-ECGs foram visualizados em um tablet da marca Apple ${ }^{\circledR}$, modelo: $\mathrm{iPad}^{\circledR} 2$, por um médico 
cardiologista com experiência na avaliação de ECGs de pacientes em tratamento intensivo.

\section{Etapa 4: Avaliação da eficácia do foto-ECG}

Utilizou-se a comparação do laudo do ECG original com o dos seus respectivos foto-ECGs (câmera Canon e celular Nokia), para verificar se a forma de obtenção e transmissão do foto-ECG interfere na leitura dos traçados, a ponto de alterar a interpretação do exame.

Dessa forma, foi considerado, para o laudo final, "Não difere", quando as análises da imagem e do respectivo ECG original coincidiram, ou "Difere", quando essas divergiram.

A estatística Kappa em malha $2 \times 2$ foi utilizada para analisar o nível de concordância entre os laudos dos ECGs em papel e dos foto-ECGs, onde um valor próximo a 1,00 representa forte nível de concordância e um valor próximo a zero representa ocorrências aleatórias, sendo a equivalência baseada na escala Landis e Koch. ${ }^{11}$ A análise estatística dos dados foi feita por meio do software on-line desenvolvido por Richard Lowry. ${ }^{12}$ Nesse caso, para viabilizar a análise, os laudos finais foram agrupados em "ECG Normal" e "ECG Alterado".

\section{RESULTADOS}

A análise dos dados demonstrou divergência em 14 dos 50 laudos (28\%) na comparação dos ECGs originais com os foto-ECGs adquiridos pela câmera Canon (Tabela 1). Dentre os casos divergentes, o cardiologista apontou dificuldade na leitura do fotoECG em 6 dos 14 casos $(42,86 \%)$.

Na comparação entre os laudos dos ECGs originais e dos foto-ECGs capturados pelo celular Nokia, verificou-se uma divergência em 13 dos 50 laudos (26\%) (Tabela 2), sendo a dificuldade na leitura do foto-ECG apontada em todos os casos (100\%).

Considerando-se os casos divergentes (14 da câmera e 13 do celular), em 12 deles o especialista atribuiu o mesmo laudo para ambos os foto-ECGs. A Figura 2 ilustra um desses casos.

A avaliação dos laudos dos ECGs originais apresentou 33 ECGs normais (66\%), 11 com hipertrofia ventricular esquerda (22\%), 4 com bloqueio de ramo direito ( $8 \%$ ) e 2 com aumento de átrio esquerdo (4\%).

O resultado Kappa demonstrou concordância considerável (Kappa $=0,356)$ entre as interpretações de foto-ECGs e ECGs em papel, apresentando o mesmo valor tanto para o celular Nokia quanto para a câmera Canon.

Tabela 1. Número e percentual (\%) de laudos divergentes entre o eletrocardiograma original e os foto-eletrocardiogramas obtidos com a câmera Canon $(\mathrm{n}=50)$.

\begin{tabular}{ccc}
\hline Laudo do ECG original & Laudo do foto-ECG Canon & n (\%) \\
\hline Normal & Bloqueio de ramo direito & $7(14)$ \\
Normal & Hipertrofia ventricular esquerda & $1(2)$ \\
Bloqueio de ramo direito & Hipertrofia ventricular esquerda & $2(4)$ \\
Hipertrofia ventricular esquerda & Normal & $2(4)$ \\
Aumento de átrio esquerdo & Normal & $2(4)$ \\
\hline
\end{tabular}

$\mathrm{ECG}=$ eletrocardiograma.

Tabela 2. Número e percentual (\%) de laudos divergentes entre o eletrocardiograma original e os foto-eletrocardiogramas obtidos com o celular Nokia $(n=50)$.

\begin{tabular}{ccc}
\hline Laudo do ECG original & Laudo do foto-ECG Nokia & n (\%) \\
\hline Normal & Bloqueio de ramo direito & $2(4)$ \\
Normal & Hipertrofia ventricular esquerda & $1(2)$ \\
Hipertrofia ventricular esquerda & Normal & $6(12)$ \\
Bloqueio de ramo direito & Normal & $2(4)$ \\
Aumento de átrio esquerdo & Normal & $2(4)$ \\
\hline
\end{tabular}

$\mathrm{ECG}=$ eletrocardiograma. 

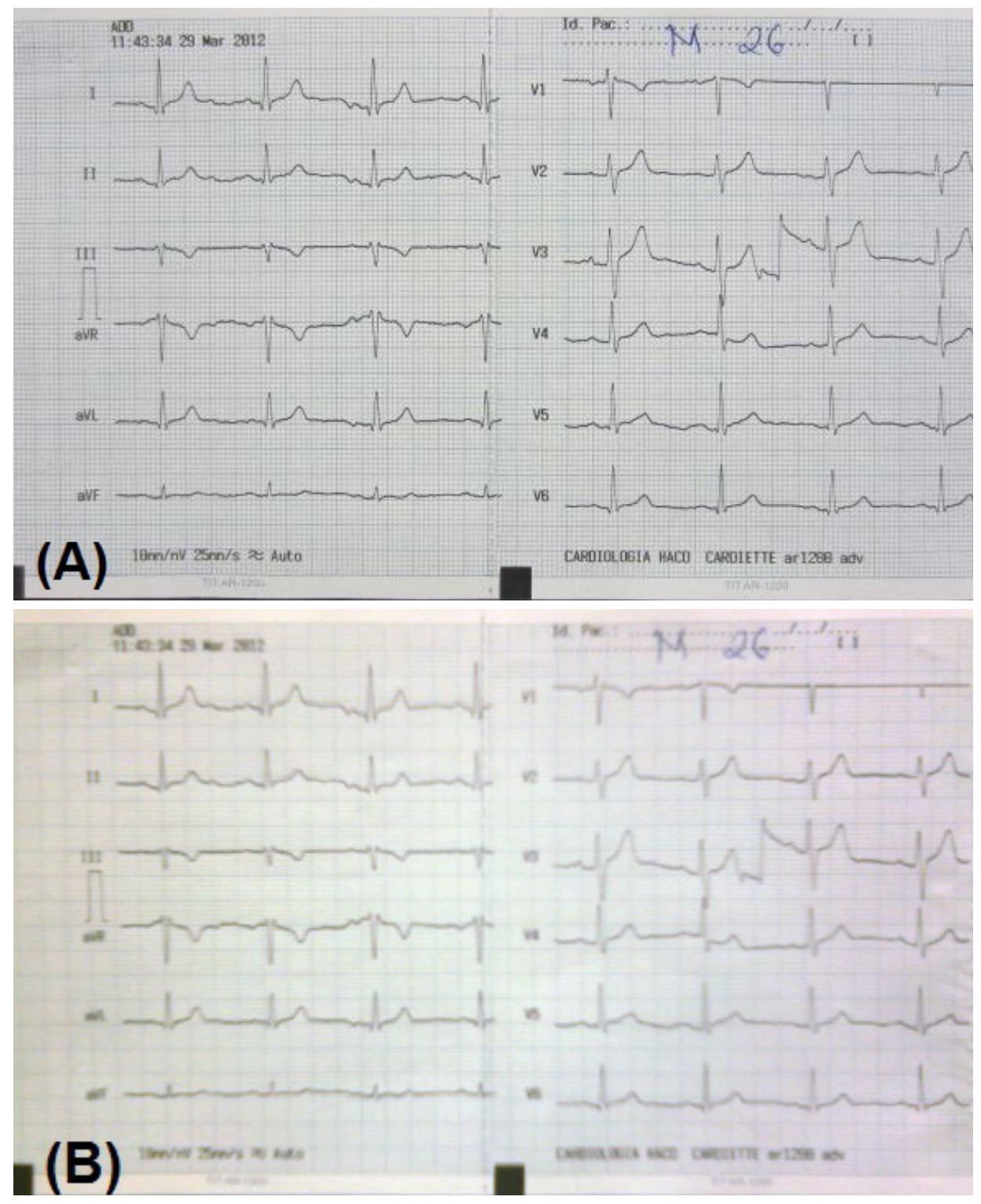

Figura 2. Comparação das imagens dos foto-eletrocardiogramas adquiridas do mesmo eletrocardiograma original: (A) pela câmera fotográfica e (B) pelo telefone celular, demonstrando melhor qualidade da imagem obtida pela câmera.

\section{DISCUSS ÃO}

A Telecardiologia, segundo Beckaman et al. ${ }^{13}$, desempenha um papel importante em permitir o diagnóstico de elevação do segmento ST no infarto agudo do miocárdio antes da internação, em casos de atendimento pré-hospitalar. Isso é de crucial importância, pois reduz o tempo para início do tratamento. Brown et al. ${ }^{14}$ demonstraram que o ECG pré-hospitalar com diagnóstico é o fator mais importante na redução do tempo porta-balão, diminuindo a mortalidade dos pacientes.
Molinari et al. ${ }^{15}$ acompanharam 100 clínicos gerais, durante um mês, os quais atenderam 456 pacientes com sintomas cardíacos e, destes, 134 eram suspeitos de ter eventos coronarianos genuínos. No entanto, através da utilização de um serviço de Telecardiologia, concluíram que 84 dos 134 pacientes com suspeição de eventos coronarianos agudos não tinham alterações eletrocardiográficas, e a hospitalização foi evitada. ${ }^{13}$

Quando comparada a outras ferramentas de Telecardiologia, as vantagens observadas na utilização do foto-ECG são dispensar o emprego de equipamentos de custo elevado e tornar a tecnologia acessível a 
praticamente toda a população. ${ }^{16}$ No presente estudo, os resultados obtidos mostram uma concordância considerável entre as análises dos ECGs originais e dos foto-ECGs adquiridos pela câmera digital $(72 \%)$ ou do celular $(74 \%)$. Resultados ainda mais expressivos foram encontrados na pesquisa de Bilgi et al., ${ }^{16}$ onde foi analisada a acurácia na interpretação de 305 ECGs obtidos em uma emergência, fotografados por um celular e enviados por sistema de mensagem multimídia ao celular de um cardiologista remoto.

Embora não tenha sido objeto desta pesquisa, outros estudos evidenciaram a redução no tempo gasto para segunda opinião formativa quando utilizado o fotoECG, sem, no entanto, avaliar a qualidade do laudo. Ohtsuka et al. ${ }^{17}$ compararam o envio de fotografias de ECG por sistema de mensagem multimídia de celular e por fax e constataram que, apesar do primeiro método diminuir em quase $50 \%$ o tempo total gasto para diagnosticar síndrome coronariana aguda a distância, a avaliação da fotografia despende o dobro de tempo comparado ao fax, justificando o fato na dificuldade em visualizar o ECG na tela do celular.

Terkelsen et al. ${ }^{18}$ e Sillesen et al. ${ }^{19}$ avaliaram o tempo economizado para iniciar uma intervenção coronária percutânea quando utilizado o foto-ECG, bem como o impacto na saúde do paciente, também encontrando resultados otimistas. Novamente, os estudos se focaram no tempo despendido e não abordaram a concordância do foto-ECG com o ECG de origem.

Esses estudos colocam o foto-ECG como uma ferramenta com forte potencial no campo da Telecardiologia, auxiliando médicos e aprimorando o atendimento prestado aos pacientes independente de sua localização. Isso o torna capaz de produzir um impacto social positivo na medida em que evita custos desnecessários do sistema de saúde e paralelamente facilita o atendimento de pacientes com doenças cardiovasculares e risco de morte elevada em centros de alta complexidade.

No presente estudo, os resultados apresentados pelo teste Kappa mostraram um nível de concordância razoável na comparação dos laudos dos ECGs originais e seus respectivos foto-ECGs. Tendo em vista que em quase todos os casos de laudos divergentes ambos os foto-ECGs obtiveram a mesma análise, acredita-se que isso se deva em parte à variabilidade de avaliação do especialista. Essa variabilidade é ainda mais evidente com a análise da indicação da dificuldade de visualização das imagens, que mesmo tendo sido presente em $100 \%$ das fotografias obtidas com o celular, contra aproximadamente $34 \%$ para câmera, levou praticamente à mesma divergência na comparação entre laudos dos ECGs originais e dos foto-ECGs.
Acredita-se que a dificuldade de visualização teve como causa a qualidade inferior das lentes fotográficas do celular e a escolha da resolução mínima disponível na máquina fotográfica. ${ }^{20}$ Entretanto, o armazenado local e o envio pela Internet dos dados gerados nessas segundas opiniões formativas virtuais constituem uma importante preocupação na viabilização de seu uso em localidades sem acesso à banda larga ou à infraestrutura de informática amplamente estabelecida. Assim, entende-se que testes-piloto, como os realizados nesse estudo, constituem uma ferramenta importante para a aceleração do processo de definição das configurações mínimas para a câmera fotográfica e para o celular, dentro dos padrões da qualidade que permitam uma concordância significativa entre laudos.

Por ainda não ter alcançado uma concordância mais elevada, entende-se que o método descrito nesse estudo deve ser aprimorado para que, então, seja utilizado como uma ferramenta de auxílio à prática clínica, através de uma segunda opinião formativa em Telecardiologia. A amostra continha somente 34\% de exames alterados, o que foi considerada uma limitação do estudo.

Assim, para estudos futuros, propõe-se a utilização de uma amostra maior e mais diversificada; a obtenção do foto-ECG com equipamentos fotográficos mais modernos; a comparação dos itens do traçado eletrocardiográfico (ritmo e frequência cardíaca, ondas, complexo, segmento e intervalos) para a melhor elucidação da dificuldade encontrada na análise e a utilização da avaliação de mais de um cardiologista para o mesmo foto-ECG.

\section{REFERÊNCIAS}

1. Pereira TP, Araujo APL, Souza CP. Um método de compressão de sinais eletrocardiográficos para uso de SMS em sistemas móveis. In: La Novena Conferencia Iberoamericana en Sistemas, Cibernética e Informática (CISCI) 2010; 2010 Jun 29 - Jul 2; Orlando, EUA. Orlando: CISCI; 2010.

2. Geremias DM, Simões PWTA, Martins PJ, Mattos MC, Casagrande RS, Salvaro RG, Goularte FB. Informática em Saúde - Segunda Opinião Formativa via Eletrocardi-ografia Digital e Informações Clínicas de um Sistema de Registro Eletrônico em Saúde. Anais SULCOMP. 2010;5(5).

3. Tachakra S, Wang XH, Istepanian RS, Song YH. Mobile e-health: the unwired evolution of telemedicine. Telemed J E Health. 2003 Fall;9(3):247-57.

4. Chandrasekhar CP, Ghosh J. Information and communication technologies and health in low income countries: the potential and the constraints. Bull World Health Organ. 2001;79(9):850-5.

5. Russomano T, Cardoso RB, Jones CR, Oliveira HW, Hütner E, Lopes MHI. e-Health Projects of the Microgravity Centre. In: Komorowska MA, Olsztynska-Janus S, editors. Biomedical Engineering, Trends, Research \& Technologies. Croácia: InTech; 2011. p. 529-50. 
6. Jones CR, Cardoso RB, Huttner E, Oliveira HW, Santos MA, Russomano T. A multidisciplinary e-Health assistance project in the Brazilian Amazon. Conference Program IADIS e-Health 2011; 2011 Jul 20-26; Roma, Itália. Roma: IADIS; 2011. p. 191-5.

7. Sood S, Mbarika V, Jugoo S, Dookhy R, Doarn CR, Prakash N, Merrell RC. What Is Telemedicine? A Collection of 104 Peer-Reviewed Perspectives and Theoretical Underpinnings. Telemed J E Health. 2007 Oct;13(5):573-90.

8. Shanit D, Cheng A, Greenbaum RA. Telecardiology: supporting the decision-making process in general practice. J Telemed Telecare. 1996;2(1):7-13.

9. Rubel P, Gouaux F, Fayn J, Assanelli D, Cuce A, Edenbrandt L, Malossi C. Towards intelligent and mobile systems for early detection and interpretation of cardiological syndromes. Comput Cardiol.. 2001;28(1):193-6.

10. Andreao RV, Filho JGP, Calvi CZ. TeleCardio - Telecardiologia a serviço de pacientes hospitalizados em domicílio. In: X Brazilian Conference in Health Informatics (CBIS); 2006 Out 14-18; Florianópolis, SC. Florianópolis: Sociedade Brasileira de Informática em Saúde; 2006.

11. Luiz RR, Costa AJL, Nadanovsky P. Epidemiologia e bioestatística na pesquisa odontológica. São Paulo: Atheneu; 2005.

12. Lowry, R. VassarStats: website for statistical computation. Poughkeepsie; 1998 [atualizado 2013; acessado em 2013 Set 12]. Disponível em: http://www.vassarstats.net/kappa. html

13. Backman W, Bendel D, Rakhit R. The telecardiology revolution: improving the management of cardiac disease in primary care. J R Soc Med. 2010 Nov;103(11): 442-6.
14. Brown JP, Mahmud E, Dunford JV, Ben-Yehuda O. Effect of prehospital 12-lead electrocardiogram on activation of the cardiac catheterization laboratory and door-to-balloon time in ST-segment elevation acute myocardial infarction. Am J Cardiol. 2008 Jan 15;101(2):158-61.

15. Molinari G, Reboa G, Frascio M, Leoncini M, Rolandi A, Balzan C, Barsotti A. The role of telecardiology in supporting the decision-making process of general practitioners during the management of patients with suspected cardiac events. J Telemed Telecare. 2002;8(2):97-101.

16. Bilgi M, Gülalp B, Erol T, Güllü H, Karagün Ö, Altay H, Müderrisoglu $\mathrm{H}$. Interpretation of electrocardiogram images sent through the mobile phone multimedia messaging service. Telemed J E Health. 2012 Mar;18(2):126-31.

17. Ohtsuka M, Uchida E, Nakajima T, Yamaguchi H, Takano $\mathrm{H}$, Komuro I. Transferring images via the wireless messaging network using camera phones shortens the time required to diagnose acute coronary syndrome. Circ J. 2007 Sept;71(9):1499-500.

18. Terkelsen CJ, Nørgaar BL, Lassen JF, Gerdes JC, Ankersen JP, Rømer F, Nielsen TT, Andersen HR. Telemedicine used for remote prehospital diagnosing in patients suspected of acute myocardial infarction. J Intern Med. 2002 Nov; 252(5):412-20.

19. Sillesen M, Sejersten M, Strange S, Nielsen SL, Lippert F, Clemmensen P. Referral of patients with ST-segment elevation acute myocardial infarction directly to the catheterization suite based on prehospital teletransmission of 12-lead electrocardiogram. J Electrocardiol. 2008 JanFeb;41(1):49-53.

20. Taylor J, Everrit PF. The optics of photography and photographic lenses. Londres: Whittaker \& co.; 1904. 